\title{
OBSTRUCTIONS AND HYPERSURFACE SECTIONS (MINIMALLY ELLIPTIC SINGULARITIES)
}

\author{
KURT BEHNKE AND JAN ARTHUR CHRISTOPHERSEN
}

\begin{abstract}
We study the obstruction space $T^{2}$ for minimally elliptic surface singularities. We apply the main lemma of our previous paper [3] which relates $T^{2}$ to deformations of hypersurface sections. To use this we classify general hypersurface sections of minimally elliptic singularities. As in the rational singularity case there is a simple formula for the minimal number of generators for $T^{2}$ as a module over the local ring. This number is in many cases (e.g. for cusps of Hilbert modular surfaces) equal to the vector space dimension of $T^{2}$.
\end{abstract}

\section{INTRODUCTION}

We continue the investigation, started in [3], of the obstruction space $T^{2}$. Recall that if $S$ is the versal base space of an isolated singularity $X$, then its tangent space at the special point is the so-called $T_{X}^{1}$. If $\operatorname{dim}_{\mathrm{C}} T_{X}^{1}=\tau$, then we may think of $S$ lying in $\left(\mathbf{C}^{\tau}, 0\right)$. Let $I_{S}$ be its ideal, $\mathbf{m}_{\tau}$ the maximal ideal at 0 in $\mathbf{C}^{\tau}$ and let $*$ denote the $\mathbf{C}$ dual of a vector space. There is an injective "obstruction map" $\left(I_{S} / \mathbf{m}_{\tau} I_{S}\right)^{*} \hookrightarrow T_{X}^{2}$. We refer, for example, to [3] and the bibliography there for definitions and properties of these modules.

This time we study minimally elliptic singularities. These are often considered the next simplest normal surface singularities, at least only the rational singularities have been more studied. If $\widetilde{X}$ is the minimal resolution of our surface singularity $X$, the conditions are that $\operatorname{dim} H^{1}\left(\widetilde{X}, \mathscr{O}_{\widetilde{X}}\right)=1$ and that $X$ is Gorenstein. Such singularities were first examined by Laufer in [9]. (See also [10].)

The results on the $T^{2}$ of minimally elliptic singularities are similar to the ones we found in the rational case in [3]. (We recall the statements from [3] in $\S 2$ so the reader may compare.) Our main result is

Theorem 6.1.1. For a minimally elliptic surface singularity of embedding dimension $n+1 \geq 5$, and $f$ the defining equation of a general hypersurface section,

1. The minimal number of generators of $T_{X}^{2}$ as an $\mathscr{O}_{X}$ module is $(n+1)(n-4) / 2$. Moreover, if $\mathbf{m}_{X}$ is the maximal ideal, then $\mathbf{m}_{X} T_{X}^{2}=$ $f T_{X}^{2}$.

Received by the editors May 1, 1990 and, in revised form, September 4, 1990.

1980 Mathematics Subject Classification (1985 Revision). Primary 14B07, 13D03, 13 D10.

Key words and phrases. Deformations of singularities, obstructions, hypersurface section, minimally elliptic singularities, degeneration of the tangent cone.

The first author was supported by a "Heisenberg Stipendium," Be 1078/1-2, of the DFG. 
2. If the exceptional curve of the blow up of the maximal ideal is reducedi.e. if the fundamental divisor on the minimal resolution is reduced on non- $(-2)$ curves-then $\operatorname{dim} T_{X}^{2}=(n+1)(n-4) / 2$.

We remark that (2) just means that all minimally elliptic singularities with fundamental divisor of the minimal resolution isomorphic to a Kodaira elliptic curve have $\operatorname{dim} T^{2}=(n+1)(n-4) / 2$. Among these are the simply elliptic singularities, the cusps, and the triangle singularities with their nonquasihomogeneous satellites.

The structure of the proof is also the same as in [3], though some of the arguments are new. Everything is built on the Main Lemma of [3] which is a formula relating $T^{2}$ to the deformations of hypersurface sections. We state the lemma in $\S 2$. We want to apply it to the general hypersurface section of a minimally elliptic singularity. It turns out that such a curve must be Gorenstein with $\delta$ invariant equal $n+1$ where $n$ is the embedding dimension of the curve. We give a classification (which may be of more general interest) of such curves in \$3-they are easily described and we call them elliptic partition curves.

We give an example of a minimally elliptic singularity with nonreduced tangent cone and $\operatorname{dim} T^{2}>(n+1)(n-4) / 2$.

\section{Hypersurface SECTIONS AND $T^{2}$}

We recall here some of the results from [3], the most important being the Main Lemma of that paper. We rely on [3] for the notation used here for the cotangent modules. We include also the classification of generic hypersurface sections of rational singularities and the main result on $T^{2}$ from that paper so the reader may compare with the corresponding statements about minimally elliptic singularities in this paper.

\subsection{Let $X$ be the germ of an analytic space of positive dimension and}

$$
f: X \rightarrow(\mathbf{C}, 0)
$$

a germ of an analytic function, i.e. $f \in \mathscr{O}_{X}$ the local ring of $X$ at 0 . We will say that $Y=f^{-1}(0)$ is a hypersurface section of $X$ if $f \in \mathbf{m}_{X}$ (the maximal ideal of germs vanishing at 0 ) and $f$ is a nonzero divisor in $\mathscr{O}_{X}$. We will from now on assume that $Y$ is an isolated singularity.

Since $f: X \rightarrow(\mathbf{C}, 0)$ is a flat deformation of $Y$, there is a map $j:(\mathbf{C}, 0) \rightarrow$ $S$ where $S$ is the base space of the versal deformation of $Y$. Define $e_{f}$ to be the dimension of the Zariski tangent space of $S$ at the generic point of the curve $j(\mathbf{C})$. If $X$ was an isolated singularity and 0 was an isolated critical point for $f$, then $f: X \rightarrow(\mathbf{C}, 0)$ is a smoothing, i.e. the generic fiber is smooth. In this case a general point $j(t)$ is smooth and $e_{f}$ is the dimension of the irreducible smoothing component containing $j(\mathbf{C}, 0)$.

Let $\mathscr{O}_{1}=\mathbf{C}\{t\}$ be the local ring of $(\mathbf{C}, 0)$ at 0 . Then $\mathscr{O}_{X}$ is an $\mathscr{O}_{1}$ module via $t \mapsto f$. There is a natural map (call it $\alpha$ ) from the relative cotangent cohomology module $T^{1}\left(\mathscr{O}_{X} / \mathscr{O}_{1} ; \mathscr{O}_{X}\right)$ to $T_{Y}^{1}$. In the proof of Wahl's conjecture on the dimension of smoothing components, Greuel and Looijenga [4] show that $e_{f}=\operatorname{dim}_{\mathbf{C}} \operatorname{Im}(\alpha)$. Using this and some standard results about the cotangent complex $[3,1.3]$ we get our Main Lemma. 
Main Lemma. If $Y=f^{-1}(0)$ is a hypersurface section of $X$ and an isolated singularity then

1. There is a long exact sequence

$$
\begin{aligned}
T^{1}\left(\mathscr{O}_{X} \mid \mathscr{O}_{1} ; \mathscr{O}_{X}\right) \stackrel{\alpha}{\rightarrow} T_{Y}^{1} \rightarrow T_{X}^{2} \stackrel{f}{\rightarrow} T_{X}^{2} \rightarrow T_{Y}^{2} & \rightarrow \cdots \\
& \cdots \rightarrow T_{X}^{i} \stackrel{f}{\rightarrow} T_{X}^{i} \rightarrow T_{Y}^{i} \rightarrow \cdots .
\end{aligned}
$$

2. $\operatorname{dim}_{\mathrm{C}}\left(T_{X}^{2} / f T_{X}^{2}\right)-\mathrm{rk}_{\mathcal{O}_{1}} T_{X}^{2}=\operatorname{dim}_{\mathbf{C}} T_{Y}^{1}-e_{f}$.

3. If $f$ is a smoothing of $Y$ then $e_{f}$ is the dimension of the smoothing component of $f$ and $\operatorname{dim}_{\mathbf{C}}\left(T_{X}^{2} / f T_{X}^{2}\right)=\operatorname{dim}_{\mathrm{C}} T_{Y}^{1}-e_{f}$.

2.2. The generic hypersurface sections of rational singularities are what we called partition curves. They are exactly the curve singularities with minimal possible delta invariant with respect to embedding dimension.

If a curve $Y$ is the union of $Y_{1}$ and $Y_{2}$, it is called decomposable if the Zariski tangent spaces of $Y_{1}$ and $Y_{2}$ have only the point 0 in common. We say that $Y$ is the wedge of $Y_{1}$ and $Y_{2}$, notation $Y=Y_{1} \vee Y_{2}$.

For $m$ a positive integer, let $Y(m) \subset \mathbf{C}^{m}$ be the monomial curve with semigroup generated by $m, m+1, \ldots, 2 m-1$ (the ordinary semigroup of genus $m-1)$. In parametric form $Y(m)$ is given by the map $\nu: \mathbf{C} \rightarrow \mathbf{C}^{m}$ sending $t$ to $\nu(t)=\left(t^{m}, \ldots, t^{2 m-1}\right)$. Let $m=m_{1}+\cdots+m_{r}$ be a partition of $m$. The partition curve associated with $m_{1}, \ldots, m_{r}$ is the wedge of monomial curves

$$
Y\left(m_{1}, \ldots, m_{r}\right)=Y\left(m_{1}\right) \vee \cdots \vee Y\left(m_{r}\right),
$$

where

$$
Y\left(m_{i}\right) \subset \mathbf{C}^{m_{i}} \subset \mathbf{C}^{m}=\mathbf{C}^{m_{1}} \oplus \cdots \oplus \mathbf{C}^{m_{r}} .
$$

For example if $m=3$ we have three partitions. $Y(1,1,1)$ is three lines in general position in $\mathbf{C}^{3}, Y(2,1)$ is a cusp in the $\left(z_{1}, z_{2}\right)$-plane and the $z_{3}$-axis, and $Y(3)$ is just the monomial curve described above.

For our purpose the main fact about partition curves is the following proposition in [3].

Proposition 2.2.1. Let $X$ be a reduced curve singularity of embedding dimension $n$.

1. The delta invariant of $X$ is at least $n-1$.

2. $\delta=n-1$ if and only if $X$ is a partition curve.

That these curves are actually the general hypersurface sections of rational singularities follows now immediately from a result of Morales, which we also need in this paper (see [3, 4.2.1], and Proposition 4.1.1 of this paper).

2.3. The main result of [3] was the following theorem on $T^{2}$ for rational singularities. For a singularity $X$, let $n+1=\operatorname{dim} \mathbf{m} / \mathbf{m}^{2}$, i.e. the embedding dimension of the singularity. For a rational singularity let $E=\bigcup E_{i}$ be the exceptional divisor of the minimal resolution and $Z$ the fundamental cycle.

Theorem 2.3.1. For a rational surface singularity $X$ of embedding dimension $n+1 \geq 4$ and $f$ the defining equation of a generic hypersurface section,

1. The minimal number of generators of $T_{X}^{2}$ as $\mathscr{O}_{X}$ module is $(n-1)(n-3)$. Moreover, if $\mathbf{m}_{X}$ is the maximal ideal, then $\mathbf{m}_{X} T_{X}^{2}=f T_{X}^{2}$. 
2. If the fundamental cycle $Z$ is reduced and if for any connected subgraph $E^{\prime} \subset E$ with $Z \cdot E^{\prime}=0$ the self-intersection number $E^{\prime} \cdot E^{\prime}=-2$ or -3 , then $\operatorname{dim}_{\mathrm{C}} T_{X}^{2}=(n-1)(n-3)$.

3. If $X$ is a quotient singularity then $\operatorname{dim}_{\mathbf{C}} T_{X}^{2}=(n-1)(n-3)$.

The condition in part (2) of the theorem means that the first blow up of the singularity has only rational double or triple points as singularities. This implies that the tangent cone has a finite-dimensional $T^{2}$ which we compute to bound $T_{X}^{2}$. The proof of Theorem 6.1.1 goes along the same lines as the proof of Theorem 2.3.1.

\section{GORENSTEIN CURVE SINGULARITIES WITH MINIMAL DELTA INVARIANT}

3.1. For an isolated Gorenstein curve singularity $(Y, 0)$ of embedding dimension $n, n \geq 3$, the delta invariant has value at least $n+1$. We show in this section that the Gorenstein curve singularities of delta invariant $n+1$ can be described rather explicitly. We call such singularities elliptic partition curves.

3.2. We begin by recalling some of the notions and results of $\S 3.1 .3$ of our paper [3]. The main tool we used there was the decomposition of curve singularities as wedges of simpler singularities together with some formulas which compute numerical invariants like, e.g., the delta invariant from the building blocks.

If $Y$ is the union of $Y_{1}$ and $Y_{2}$ with no component in common, and $I_{k}$ is the ideal of $Y_{k}$ in an ambient $\mathbf{C}^{n}, k=1,2$, the intersection number $i\left(Y_{1}, Y_{2}\right)=$ $\operatorname{dim}_{\mathbf{C}} \mathscr{O}_{\mathbf{C}^{n}, 0} /\left(I_{1}+I_{2}\right)$. We need the assertions of three lemmas from [3, §3.1.3].

Lemma 3.2.1. Let $Y=Y_{1} \cup Y_{2}$ be a union of two curve singularities.

1. $Y=Y_{1} \vee Y_{2}$ if and only if $i\left(Y_{1}, Y_{2}\right)=1(c f .1 .2)$.

2. If $\delta, \delta_{1}, \delta_{2}$ are the delta invariants of $Y, Y_{1}$, and $Y_{2}$,

$$
\delta=\delta_{1}+\delta_{2}+i\left(Y_{1}, Y_{2}\right) .
$$

3. In particular if $Y$ has $r$ irreducible components $Y_{1}, \ldots, Y_{r}$,

$$
\delta \geq \delta_{1}+\cdots+\delta_{r}+r-1
$$

with equality if and only if $Y=Y_{1} \vee \cdots \vee Y_{r}$.

4. For $Y$ a wedge of $Y_{1}$ and $Y_{2}$, the Cohen-Macaulay types $t, t_{1}$, and $t_{2}$ are linked by the formula $t=t_{1}+t_{2}+1$. Here we set $t_{i}=0$ formally if $Y_{i}$ is a smooth branch. In particular the only Gorenstein curve singularity which is a wedge is the ordinary plane double point.

3.3. Let $n \geq 2$ be an integer. We are going to define a set of curve singularities $Y_{\mathbf{p}}, \mathbf{p}=\left(p_{1}, \ldots, p_{r}\right)$ a partition of $n+1$, Gorenstein and with embedding dimension $n$ and delta invariant $\delta=n+1$. We call $Y_{\mathbf{p}}$ the elliptic partition curve associated with the partition $\mathbf{p}$.

If $\mathbf{p}=(n+1)$ let $Y_{\mathbf{p}}$ be the monomial curve with semigroup

$$
\{0, n+1, \ldots, 2 n, 2 n+2, \ldots\},
$$

i.e., with gap set $\{1,2, \ldots, n, 2 n+1\}$. 
For $\mathbf{p}=\left(p_{1}, \ldots, p_{r}\right)$ with $r \geq 2$ the curve $Y_{\mathbf{p}}$ is reducible, with irreducible components $Y\left(p_{i}\right)$ (cf. §2.2). Choose linear subspaces $V_{i} \cong \mathbf{C}^{p_{i}}$ in $\mathbf{C}^{n}$ in general position, and embed the corresponding monomial curve $Y_{i}$ into $V_{i}$. Let $Y_{\mathbf{p}}=Y_{1} \cup \cdots \cup Y_{r}$.

More explicitly one can choose a linear embedding

$$
\mathbf{C}^{p_{1}} \oplus \cdots \oplus \mathbf{C}^{p_{r-1}} \hookrightarrow \mathbf{C}^{n} .
$$

Denote by $Y^{\prime}$ the wedge

$$
Y_{1} \vee \cdots \vee Y_{r-1} \subset \mathbf{C}^{p_{1}} \oplus \cdots \oplus \mathbf{C}^{p_{r-1}}
$$

and put $Y_{r} \subset \mathbf{C}^{p_{r}}$ in general position relative to $\mathbf{C}^{p_{1}}, \ldots, \mathbf{C}^{p_{r-1}}$. General position means that the projection of the tangent line of $Y_{r}$ to $\mathrm{C}^{p_{i}}$ does not map into the osculating hyperplane of $Y_{i}$, i.e. the hyperplane generated by the first $p_{i}-1$ basis vectors in the standard representation of $Y_{i}, i=1, \ldots, r-1$.

Obviously $Y_{\mathbf{p}}$ has embedding dimension $n$. For the delta invariant observe that the wedge $Y^{\prime}$ has $\delta^{\prime}=\sum_{i=1}^{r-1}\left(p_{i}-1\right)+r-2$ by [3]. The delta invariant of the last component is $p_{r}-1$, and the intersection number $i\left(Y^{\prime}, Y_{r}\right)=2$ as can easily be seen from a parametrization we display in the proof of Proposition 3.4.1.

Examples. The most immediate examples are for $n=2$ the ordinary triple point $(\mathbf{p}=(1,1,1))$, the union of a plane cusp and a line, different from the tangent line of the cusp $(\mathbf{p}=(2,1))$, and the plane curve singularity with equation $y^{3}=x^{4}$.

If $n=4$, and the partition is $(3,2)$ then we have equations

$$
x_{1} x_{3}, \quad x_{1} x_{4}, \quad x_{2} x_{3}-x_{4}^{2}, \quad x_{2} x_{4}-x_{3}^{3}, \quad x_{2}^{2}-x_{3}^{2} x_{4}-x_{1}^{3} .
$$

Observe that these are the Pfaffians of the alternating $(5 \times 5)$-matrix

$$
\left(\begin{array}{ccccc}
0 & -x_{1}^{2} & x_{3}^{2} & -x_{2} & x_{4} \\
x_{1}^{2} & 0 & -x_{2} & x_{4} & -x_{3} \\
-x_{3}^{2} & x_{2} & 0 & x_{1} & 0 \\
x_{2} & -x_{4} & -x_{1} & 0 & 0 \\
-x_{4} & x_{3} & 0 & 0 & 0
\end{array}\right) .
$$

For general $n$ the singularity of $n+1$ lines through the origin in complex $n$ space is the elliptic partition curve which corresponds to $n+1=1+\cdots+1$. The ideal of the monomial curve corresponding to the partition $(n+1)$ is generated (not minimally though) by the $(2 \times 2)$-minors of the "array"

$$
\left|\begin{array}{cccccc}
x_{1} & x_{2} & \cdots & x_{n-3} & x_{n-1} & x_{n-2} \\
x_{2} & x_{3} & \cdots & x_{n-2} & x_{n} & x_{n-1} \\
x_{4} & x_{5} & \cdots & x_{n} & x_{1}^{2} &
\end{array}\right|
$$

It is easy to see from an explicit parametrization, say, that all the elliptic partition curves are quasihomogeneous curve singularities. Moreover it will become clear in the proof of Proposition 3.4.1 that elliptic partition curves only depend on the partition, i.e. that there are no moduli. 
3.4. The main result of this section is

Proposition 3.4.1. An isolated Gorenstein curve singularity of embedding dimension $n$ and minimal delta invariant $n+1$ is isomorphic to an elliptic partition curve $Y_{\mathbf{p}}$ for some partition $\mathbf{p}=\left(p_{1}, \ldots, p_{r}\right)$ of $n+1$.

Proof. Let $Y=Y_{1} \cup \cdots \cup Y_{r}$ be the decomposition into irreducible components. Then by Lemma 3.2.1,

$$
n+1=\delta(Y)=\sum_{i=1}^{r} \delta\left(Y_{i}\right)+\sum_{i=1}^{r-1} i\left(Y_{1} \cup \cdots \cup Y_{i}, Y_{i+1}\right) .
$$

If we denote the embedding dimension of the branch $Y_{i}$ by $n_{i}$ we have $\delta\left(Y_{i}\right) \geq$ $n_{i}-1$. Also $\sum_{i=1}^{r} n_{i} \geq n+1$, because $Y$ is not a wedge (we exclude the trivial case of an ordinary double point here). All the intersection numbers in (1) are at least one, and they cannot be all equal to one, again because $Y$ is not a wedge. We conclude that either $Y$ is irreducible or

$$
\sum_{i=1}^{r-1}\left(i\left(Y_{1} \cup \cdots \cup Y_{i}, Y_{i+1}\right)-1\right)=1 .
$$

This means in the last case that $Y_{i}$ is the monomial curve with semigroup $\left\{0, n_{i}, \ldots, 2 n_{i}-1, \ldots\right\}[3,3.3 .1]$.

Case 1. $Y$ is irreducible. It follows from the Gorenstein property of $Y$ that the value semigroup is symmetric (cf. [8]). The only possibility is

$$
\{0, n+1, n+2, \ldots, 2 n, 2 n+2,2 n+3, \ldots\}
$$

and as in (3.3.1) of [3] we see that $Y$ is the monomial curve with the given semigroup.

Case 2. Assume now that $Y$ has at least two branches. Then there is one intersection number in (1) equal to 2 . Necessarily it must be the last one. We shall investigate how in this case the curve $Y$ can be built from its components.

Let $n: \widehat{Y} \rightarrow Y$ be the normalization map, and let $\widehat{Y}_{i}$ be the smooth curve mapped onto the branch $Y_{i}$ under $n$. We can find parameters $t_{1}, \ldots, t_{r}$ of $Y_{1}, \ldots, Y_{r}$, local coordinates

$x_{1,1}, \ldots, x_{1, n_{1}}, x_{2,1}, \ldots, x_{2, n_{2}}, \ldots, x_{r-1,1}, \ldots, x_{r-1, n_{r-1}}, x_{r, 1}, \ldots, x_{r, n_{r}-1}$ around 0 in $\mathbf{C}^{n}$, and holomorphic power series

$$
f_{1,1}, \ldots, f_{1, n_{1}}, \ldots, f_{r-1,1}, \ldots, f_{r-1, n_{r-1}}, f_{r, 1}, \ldots, f_{r, n_{r}-1}
$$

such that the map $n$ looks as indicated in Table 1 on page 182.

Since the branch $Y_{r}$ is a monomial curve with ordinary semigroup $\left\{0, n_{r}\right.$, $\left.n_{r}+1, \ldots, 2 n_{r}-1, \ldots\right\}$ the orders of the power series $f_{i, j}\left(t_{r}\right)$ are at least $n_{r}$. The intersection number $i\left(Y_{1} \cup \cdots \cup Y_{r-1}, Y_{r}\right)=2$ translates into the fact that the orders of the power series $f_{r, 1}, \ldots, f_{r, n_{r}-1}$ leave out exactly one value in the range $n_{r}, \ldots, 2 n_{r}-1$. To see this observe that the ideal of functions on $\mathbf{C}^{n}$ which vanish on $Y_{1} \cup \cdots \cup Y_{r-1}$ is generated by the coordinates $x_{r, 1}, \ldots, x_{r, n_{r}-1}$ and a set of power series in the other coordinates which have quadratic initial forms. So

$$
\begin{aligned}
& i\left(Y_{1} \cup \cdots \cup Y_{r-1}, Y_{r}\right) \\
& \quad \geq \operatorname{dim}_{\mathbf{C}}\left(\mathbf{C}\left\{f_{i, j}\right\} /\left(f_{r, 1}, \ldots, f_{r, n_{r-1}}\right)+\left(f_{1,1}, \ldots, f_{r-1, r_{r-1}}\right)^{2}\right)
\end{aligned}
$$

whence the claim. 
Local duality tells us which of the orders is actually missing. The module of Rosenlicht differentials

$$
\omega_{Y}=\left\{\eta \in \Omega_{\widehat{Y}}^{1}(*) \mid \operatorname{res}(f \eta)=0 \forall f \in \mathscr{O}_{Y, 0}\right\},
$$

where $\Omega_{\widehat{Y}}^{1}(*)$ means meromorphic 1 -forms with poles at most at the distinguished points of $\widehat{Y}_{i}$, and $\operatorname{res}(f \eta)$ is the sum of all residues in the ordinary sense, is the dualizing module of $Y$. It is a cyclic module because $Y$ is Gorenstein, and the length of the factor space $\left(\omega_{Y} / n_{*} \Omega_{Y}^{1}\right)$ is $\delta_{Y}=n+1$ by local duality.

We can at once write down $n$ independent meromorphic elements of $\omega_{Y}$ :

$$
\begin{gathered}
\frac{d t_{1}}{t_{1}^{n_{1}}}, \ldots, \frac{d t_{1}}{t_{1}^{2}}, \ldots, \frac{d t_{r}}{t_{r}^{n_{r}}}, \ldots, \frac{d t_{r}}{t_{r}^{2}}, \\
\frac{d t_{1}}{t_{1}}-\frac{d t_{2}}{t_{2}}, \ldots, \frac{d t_{r-1}}{t_{r-1}}-\frac{d t_{r}}{t_{r}} .
\end{gathered}
$$

The missing form $\eta_{0}$ must be a generator of $\omega_{Y}$, and all its multiples have to be linear combinations of these meromorphic forms and holomorphic differentials. We conclude that the order of pole of $\eta_{0}$ in $t_{i}$ is $2 n_{i}$, so that we can assume

$$
\eta_{0}=\sum_{i=1}^{r} \sum_{\nu=1}^{n_{i}} a_{i, \nu} \frac{d t_{i}}{t_{i}^{n_{i}+\nu}}, \quad a_{i, n_{i}} \neq 0 .
$$

Multiplication by $x_{r, 1}, \ldots, x_{r, n_{r}-1}$ gives us a differential form

$$
f_{r, j}\left(t_{r}\right) \sum_{\nu=1}^{n_{r}} a_{r, \nu} \frac{d t_{r}}{t_{r}^{\nu+n_{r}}}
$$

which must have vanishing residue. So we see that the power series $f_{r, i}$ have orders $n_{r}, \ldots, 2 n_{r}-2$. After an obvious coordinate change we arrive at the following representation of the normalization map (Table 2).

From this representation it is not difficult to compute the generator $\eta_{0}$ of $\omega_{Y}:$

$$
\eta_{0}=-\sum_{i=1}^{r-1} \sum_{\nu=1}^{n_{i}} b_{i, \nu} \frac{d t_{i}}{t_{i}^{\nu+n_{i}}}+\frac{d t_{r}}{t_{r}^{2 n_{r}}} .
$$

In particular the coefficient $b_{i, n_{i}} \neq 0$, and we have proved that the tangent line of the component $Y_{r}$ does not project into an osculating hyperplane of one of the other branches $Y_{i}, i \leq r-1$, which are defined by $y_{i, n_{i}}=0$. In the last step of the proof we want to show that $Y$ is actually isomorphic to a standard elliptic partition curve. In particular this would imply that elliptic partition curves have no moduli. To this end we assume that we only have two components: an ordinary monomial curve and a line, not in the osculating hyperplane. Indeed, the projection to $\mathrm{C}^{n_{1}+\cdots+n_{r-1}}$ of the last component $Y_{r}$ is a line, and the coordinate transformations still to come will only affect one of the coordinate blocks $y_{i, 1}, \ldots, y_{i, n_{i}}$ at a time.

Let the two components of $Y$ be parametrized by

$$
\begin{aligned}
t \mapsto\left(y_{1}, \ldots, y_{n}\right) & =\left(t^{n}, \ldots, t^{2 n-1}\right), \\
s & \mapsto\left(y_{1}, \ldots, y_{n}\right)=\left(c_{1} s, \ldots, c_{n} s\right), \quad c_{n} \neq 0 .
\end{aligned}
$$



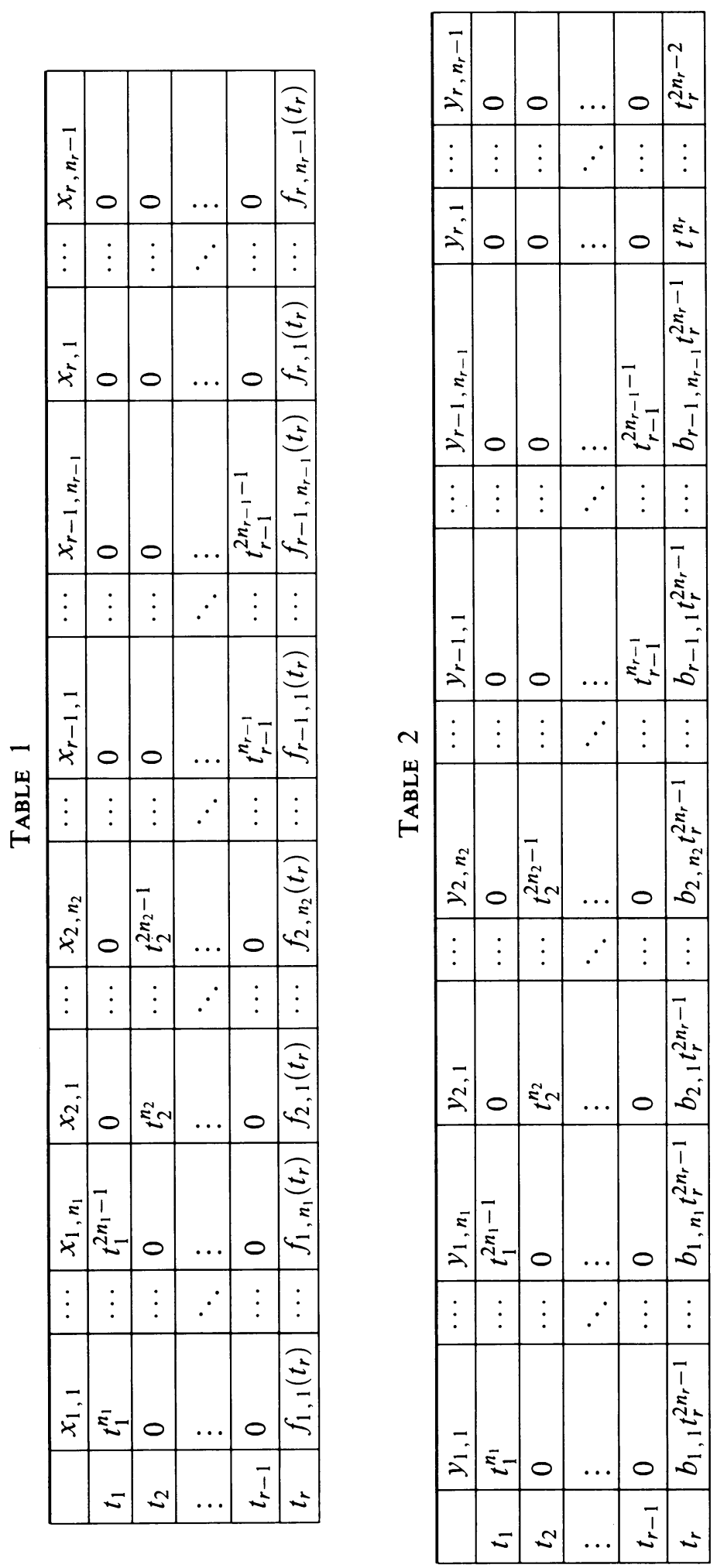
We want to show that we can perform coordinate transformations both in $s, t$ and in $y_{1}, \ldots, y_{n}$ such that the parameterization in the new variables is

$$
\begin{aligned}
& t_{1} \mapsto\left(y_{1}^{\prime}, \ldots, y_{n}^{\prime}\right)=\left(t_{1}^{n}, \ldots, t_{1}^{2 n-1}\right), \\
& s_{1} \mapsto\left(y_{1}^{\prime}, \ldots, y_{n}^{\prime}\right)=\left(0, \ldots, 0, s_{1}\right) .
\end{aligned}
$$

Isolated curve singularities are finitely determined in the sense that if the parametrizations of the components agree up to a certain finite order then two given curves are actually isomorphic. For example, by [6, Theorem B] it is sufficient to check an isomorphism modulo $\mathbf{m}^{j+1}$, where $j \geq 3 \delta+1$.

First let $y_{i}^{\prime}=y_{i}-c_{i} c_{n}^{-1} y_{n}, \quad i=1, \ldots, n-1$, and $y_{n}^{\prime}=y_{n}$. Then in $y^{\prime}$-coordinates the line is parametrized by $s \mapsto\left(0, \ldots, 0, c_{n} s\right)$, and rescaling $s_{1}=c_{n} s$ gives us what we want it to be.

The first component now has the parametrization

$$
t \mapsto\left(y_{1}^{\prime}, \ldots, y_{n}^{\prime}\right)=\left(t^{n}-c_{1} t^{2 n-1}, \ldots, t^{2 n-2}-c_{n-1} t^{2 n-1}, t^{2 n-1}\right) .
$$

We apply a parameter transformation of the form

$$
t=p_{1} t^{\prime}+p_{2}\left(t^{\prime}\right)^{2}+\cdots+p_{n}\left(t^{\prime}\right)^{n}, \quad p_{1} \neq 0,
$$

to kill the term of order $2 n-1$ in $y_{1}^{\prime}\left(t^{\prime}\right), \ldots, y_{n-1}^{\prime}\left(t^{\prime}\right)$. To find such a transformation observe that for the vanishing of the coefficient of $\left(t^{\prime}\right)^{2 n-1}$ in $y_{i}^{\prime}\left(t^{\prime}\right)$ we get $(n-1)$ quasihomogeneous equations in the $n$ variables $p_{1}, \ldots, p_{n}$. For example the coefficient of $t^{\prime}$ in $y_{n-1}^{\prime}$ is

$$
p_{1}^{2 n-3}\left((2 n-2) p_{2}-c_{n-1} p_{1}^{2}\right)=0 \text {. }
$$

So we can now assume that the power series (which are in fact polynomials) $y_{1}^{\prime}\left(t^{\prime}\right), \ldots, y_{n-1}\left(t^{\prime}\right)$ have orders $n, \ldots, 2 n-2$, and zero coefficient at $\left(t^{\prime}\right)^{2 n-1}$. Next we eliminate all the terms of order $n+i, \ldots, 2 n-2$ from $y_{i}^{\prime}$ by a routine process. We begin by adding a suitable multiple of $y_{n-1}^{\prime}$ to $y_{n-2}^{\prime}$, in order to kill the coefficient of $\left(t^{\prime}\right)^{2 n-2}$ in $y_{n-2}^{\prime}$. A linear combination of $y_{n-1}^{\prime}$ and $y_{n-2}^{\prime}$ added to $y_{n-3}^{\prime}$ annihilates its terms of order $2 n-2$ and $2 n-3$. We keep on doing this until we arrive at coordinates $y_{1}^{\prime \prime}, \ldots, y_{n-1}^{\prime \prime}, y_{n}^{\prime \prime}=y_{n}$ such that $y_{i}^{\prime \prime}\left(t^{\prime}\right)=\left(t^{\prime}\right)^{n+i-1}+$ terms of order at least $2 n$.

Each integer bigger than $2 n-1$ can be represented as a nonnegative linear combination of $n, \ldots, 2 n-2$. Hence by adding monomials in $y_{k}^{\prime \prime}, k=$ $1, \ldots, n-1$, to $y_{i}^{\prime \prime}, i=1, \ldots, n$, we can substitute any term in the power series $y_{i}^{\prime \prime}\left(t^{\prime}\right)$ by a polynomial which has higher order as a power series. After finitely many steps there are no terms left modulo $\mathbf{m}^{3 \delta+1}$, and by a final change of coordinates we get what we want.

3.5. Recall that the partition curve corresponding to $n+1=n_{1}+\cdots+n_{r}$ is just the wedge of the corresponding monomial curves in $\mathbf{C}^{n+1}$. We have thus shown that the image of a partition curve $Y\left(n_{1}, \ldots, n_{r}\right) \hookrightarrow \mathbf{C}^{n+1}$ under a general linear projection $\mathbf{C}^{n+1} \rightarrow \mathbf{C}^{n}$ is an elliptic partition curve and that every elliptic partition curve arises that way.

\section{Elliptic PARTITION CURVES AS HYPERSURFACE SECTIONS OF MINIMALLY ELLIPTIC SINGULARITIES}

4.1. We show that the general hypersurface sections of minimally elliptic surface singularities are elliptic partition curves. Moreover, all elliptic partition 
curves appear as special hypersurface sections of cones over elliptic curves (simply elliptic singularities). This last fact is needed for the proof of Theorem 6.1.1.

Proposition 4.1.1. Let $(X, 0)$ be a minimally elliptic surface singularity of multiplicity $n+1 \geq 3$. The general hypersurface section $(Y, 0)$ of $(X, 0)$ is an elliptic partition curve, associated with a partition $n+1=n_{1}+\cdots+n_{r}$.

Proof. By [3, Corollary 4.2.2] the delta invariant of $(Y, 0)$ is $\delta=-Z^{2}=n+1$ (observe the shift from $n$ to $n+1$ in the notation), $Z$ the fundamental cycle of the minimal resolution, say. Also $(Y, 0)$ is clearly Gorenstein. So the result follows from the previous section.

Remark. We do not discuss the case of double point elliptic singularities here. The reader may verify that all general hypersurface sections of minimally elliptic singularities with $Z^{2}=-1$ or -2 are

1. ordinary cusps (for $Z^{2}=-1$ ),

2. tacnodes and higher cusps with equation $y^{2}=x^{5}$ (for $Z^{2}=-2$ ).

As in the rational case one can say a bit more about the partition which defines $(Y, 0)$.

Proposition 4.1.2. Let $\pi: \widetilde{X} \rightarrow X$ be the minimal resolution of $X$, let $E=$ $\bigcup_{i=1}^{k} E_{i}$ be the decomposition of the exceptional curve into irreducible components, and let $Z=\sum_{i=1}^{k} n_{i} E_{i}$ be the fundamental cycle. Let $r_{i}=-E_{i} E_{i}-2$. Then the partition for $(Y, 0)$ is

$$
(\underbrace{n_{1}, \ldots, n_{1}}_{r_{1}} \underbrace{n_{2}, \ldots, n_{2}}_{r_{2}}, \ldots, \underbrace{n_{k}, \ldots, n_{k}}_{r_{k}})
$$

where of course the $n_{i}$ with $r_{i}=0\left(E_{i}^{2}=-2\right)$ are omitted.

Proof. The argument is the same as in $[3,4.3 .1]$ except that we know even a bit more here: The fundamental cycle is an anticanonical cycle on $\widetilde{X}$, and hence by adjunction

$$
-Z \cdot E_{i}=-2-E_{i} \cdot E_{i}
$$

\subsection{Example.}

1. For all minimally elliptic singularities which have a fundamental cycle which is a Kodaira elliptic curve, the general hypersurface section is isomorphic to the union of $n+1$ lines in $\mathbf{C}^{n}$.

2. The partition $(2,1)$ of 3 shows up for

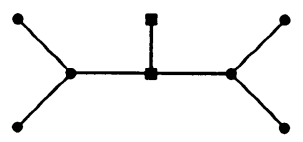

$$
\begin{aligned}
& \mathbf{\square} \cong \mathbf{P}^{1} \text {, self-int. }-3 \\
& \bullet \cong \mathbf{P}^{1} \text {, self-int. }-2
\end{aligned}
$$

The reader may check this using the equation

$$
x^{2} y+\left(z^{2}+y^{3}\right)\left((z+y)^{2}+y^{3}\right)=0 .
$$

3. To find the partition (3) look e.g. at 


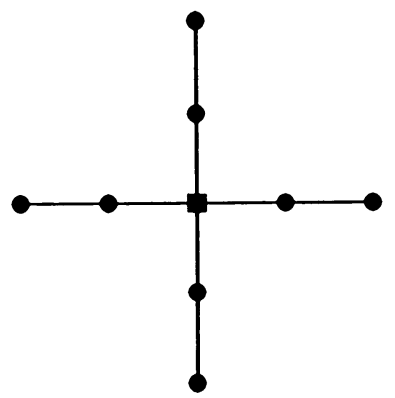

with equation $z^{3}=x^{4}+y^{4}$.

4.3. In the case of rational surface singularities all the possible hypersurface sections-the partition curves-actually show up. We do not know if the corresponding fact is true for minimally elliptic singularities.

We prove here that each elliptic partition curve can be obtained as a special hypersurface section of a cone over an elliptic curve, embedded in $\mathbf{P}^{n}$ by a complete linear system of degree $n+1$. We show even more: there is a flat $n$ parameter family of hypersurface sections on such a simply elliptic singularity, containing all the elliptic partition curves of multiplicity $n+1$. This will enable us to compute their deformation invariants.

Let for this subsection $X$ be a fixed simply elliptic singularity of both multiplicity and embedding dimension $n+1 \geq 3$. Let $\pi: \widetilde{X} \rightarrow X$ be the minimal resolution, and denote the exceptional elliptic curve by $E$. Let $L$ be the normal bundle of $E$ in $\widetilde{X}$, of degree $-(n+1)$, and choose a divisor $D_{L}$ for $L^{*}$. We identify the abelian group $E$ with $\mathrm{Pic}^{n+1} E$ by means of $D_{L}$. In particular we choose the origin in $E$ such that the sum of the points in $D_{L}$ is 0 .

We have to deal with families of divisors on $\widetilde{X} \times T, T$ a parameter space.

Lemma 4.3.1. Let $T$ be a contractible Stein space (e.g., a ball) and let $\mathscr{D}$ be an effective divisor on $\tilde{X} \times T$. Write

$$
\mathscr{D}=l \cdot(E \times T)+\sum_{\nu=1}^{k} l_{\nu} \Delta^{(\nu)}, \quad l, l_{\nu} \in \mathbf{Z},
$$

where $l \cdot(E \times T)$ is the vertical part of $\mathscr{D}$, proper over $T$. Possibly after shrinking $T$ there exists an open neighborhood $U$ of $E \times T$ in $\widetilde{X} \times T$ and a holomorphic function on $U$ with divisor $\mathscr{D} \cap U$ if and only if

1. for each $t \in T \quad l(n+1)=\sum_{\nu} l_{\nu} i\left(\Delta^{(\nu)}, E \times\{t\}\right)$,

2. for each $t \in T$ the sum

$$
\sum_{\nu=1}^{k} l_{\nu} p_{\nu}=0, \quad\left\{p_{\nu}\right\}=\Delta_{t}^{(\nu)} \cap E \times\{t\},
$$

in the group $E$.

Proof. There is a commutative diagram with exact rows and vertical isomorphisms as indicated 


$$
\begin{aligned}
& H^{1}(\tilde{X} \times T, \mathbf{Z}) \quad \rightarrow \quad H^{1}(\tilde{X} \times T, \mathscr{O}) \\
& \downarrow \cong \quad \downarrow \cong \\
& H^{1}(E \times T, \mathbf{Z}) \quad \rightarrow \quad H^{1}(E \times T, \mathscr{O}) \\
& \stackrel{\exp }{\rightarrow} H^{1}\left(\tilde{X} \times T, \mathscr{O}^{*}\right) \stackrel{c}{\rightarrow} H^{2}(\tilde{X} \times T, \mathbf{Z}) \rightarrow 0 \\
& \downarrow \cong \\
& \stackrel{\exp }{\rightarrow} H^{1}\left(E \times T, \mathscr{O}^{*}\right) \stackrel{c}{\rightarrow} H^{2}(E \times T, \mathbf{Z}) \rightarrow 0 .
\end{aligned}
$$

In particular, the remaining vertical map is an isomorphism, and for $\mathscr{D}$ to be principal on $\tilde{X} \times T$ it is sufficient that the restriction to $E \times T$ is principal. Assume (1) and (2) hold. By (1) the image under the Chern homomorphism $c(\mathscr{D} \cap(E \times T))=0$, so we have to check that the restriction of $\mathscr{D}$ to $E$ is trivial in $\mathrm{Pic}^{0}(E \times T)$. This can be done fibre by fibre (see, e.g., [5, Chapter III, Example 12.4]), and the claim follows.

The function with divisor $\mathscr{D}$ is now constructed using the theorem on holomorphic functions as in [1, Theorem 4].

4.4. We are going to construct such a divisor on $\tilde{X} \times T$, which then by Lemma 4.3.1 contracts to a family of hypersurface sections on the simply elliptic singularity $X$. Let $P_{0}$ be the sum of the points of $D_{L}$ on the elliptic curve $E$. Choose a trivializing chart $U$ for $L$ around $P_{0}$ with local coordinates $x, y$ such that $P_{0}=\{x=y=0\}$, and $E=\{y=0\}$ locally. Let $t_{0}, \ldots, t_{n}$ be local coordinates in $\mathbf{C}^{n+1}$, and let $\rho$ be so small that for $\left\|\left(t_{0}, \ldots, t_{n}\right)\right\|<2 \rho$ the points $\left(t_{i}, 0\right)$ are still in $U$. Let

$$
T=\left\{t=\left(t_{0}, \ldots, t_{n}\right) \mid \sum_{i=0}^{n} t_{i}=0,\|t\|<\rho\right\}
$$

and let $\mathscr{D} \subset U \times T$ be the set

$$
(E \cap U) \times T \cup\left\{(x, y, t) \in U \times T \mid y=\prod_{i=0}^{n}\left(t-t_{i}\right)\right\} .
$$

After restricting $\tilde{X}$ to a smaller neighborhood of $E$, if necessary, the divisor $\mathscr{D}$ defines a divisor on $\tilde{X} \times T$ which we also denote by $\mathscr{D}$. By construction, $\mathscr{D}$ fits the hypotheses of Lemma 4.3.1, i.e. there is a holomorphic function $\tilde{f}(x, t)$ on $\tilde{X} \times T$ with divisor $\mathscr{D}$, and there is a holomorphic function on $X \times T$, denoted by $f$, with pullback $\tilde{f}$.

Proposition 4.4.1. 1. All the elliptic partition curves associated with partitions $n+1=n_{1}+\cdots+n_{r}$ can be obtained as hypersurface sections of a simply elliptic singularity of degree $n+1 \quad(n \geq 2)$.

2. There is a flat and $\delta$-constant family of hypersurface sections $Y_{t}, t \in T$, of the simply elliptic singularity $X$. All elliptic partition curves of multiplicity $n+1$ occur in this family. The most special fibre $Y_{0}$ is the monomial curve. An elliptic partition curve $Y_{t^{\prime}}$ is adjacent to $Y_{t}$ if and only if the partition $\left(n_{1}^{\prime}, \ldots, n_{r^{\prime}}^{\prime}\right)$ is smaller than $\left(n_{1}, \ldots, n_{r}\right)$ in the sense that after renumbering, $n_{1}=n_{1}^{\prime}+\cdots+$ $n_{s_{1}}^{\prime}, \ldots, n_{r}=n_{s_{r-1}+1}^{\prime}+\cdots+n_{r^{\prime}}^{\prime}$

Proof. (1) is obviously a consequence of (2). The family of hypersurfaces in (2) is given by the function $f_{t}(x)=f(x, t)$ on $X \times T$. The fibres of $f_{t}$ are 
easy to analyze: if the polynomial $y=\prod_{i=0}^{n}\left(x-t_{i}\right)$ has $r$ distinct zeroes with multiplicities $n_{1}, \ldots, n_{r}$ then $f_{t}^{-1}(0)$ is an elliptic partition curve of type $\left(n_{1}, \ldots, n_{r}\right)$. The assertions about adjacencies follow immediately.

\section{ANNIHILATION OF $T^{2}$ FOR ELLIPTIC PARTITION CURVES}

5.1. In this section we compute the $T^{2}$ of elliptic partition curves. The main finding is that they are all annihilated by the maximal ideals of their local rings. The proof of this assertion is rather indirect though: its ingredients are the study of the cotangent cohomology of the Gorenstein fat point which is the general hypersurface section of such a curve singularity, and a computation of $T^{2}$ of the monomial curve with semigroup $\{0, n+1, \ldots, 2 n, 2 n+2, \ldots\}$.

5.2. The general hypersurface section of an elliptic partition curve is a Gorenstein fat point of multiplicity $r+2=n+1$ in $r=n-1$ dimensional space (cf. [11]). Its algebra structure is given by a basis $e_{0}, e_{1}, \ldots, e_{r}, e_{r+1}$, where $e_{0}$ is the unit element, and the only nontrivial products are

$$
e_{1}^{2}=\cdots=e_{r}^{2}=e_{r+1} .
$$

A finite free resolution of this algebra and its space of infinitesimal deformations have been provided in [2]. We recall some data of the minimal free resolution over the polynomial ring in $r$ variables $x_{1}, \ldots, x_{r}$. Of particular interest is the natural grading on this complex.

Proposition 5.2.1. The minimal Artinian Gorenstein algebra of embedding dimension $r$ has a pure minimal free resolution. The generators of the ith module of syzygies are in degree $i+1$ for $1 \leq i \leq r-1$, and in degree $r+2$ for $i=r$. The ith module of syzygies is minimally generated by

$$
b_{i}=\frac{i(r-i)}{r+1}\left(\begin{array}{c}
r+2 \\
i+1
\end{array}\right)
$$

elements $\left(i \leq r-1, b_{r}=1\right)$.

In particular there are $(r-1)(r+2) / 2$ quadratic equations for the singularity with $r(r+2)(r-2) / 3$ linear relations $(r \geq 3)$ and $r(r-1)(r-3)(r+2) / 8$ linear second syzygies $(r \geq 4)$.

5.3. The next result gives the first 3 cotangent cohomology groups of this algebra.

Proposition 5.3.1. The minimal Artinian Gorenstein algebra $A$ of embedding dimension $r \geq 3$ has

1. $\operatorname{dim}_{\mathrm{C}} T_{A}^{0}=\left(r^{2}+r+2\right) / 2$.

2. $\operatorname{dim}_{\mathrm{C}} T_{A}^{1}=r(r-1)(r+4) / 6$, and $T_{A}^{1}$ is concentrated in degree -1 .

3. $\operatorname{dim}_{C} T_{A}^{2}=r(r+1)(r+2)(r-3) / 12$, and $T_{A}^{2}$ is concentrated in degree -2 .

Proof. We give a proof for (3). We show that $\left(T_{A}^{2}\right)_{d}=0$ for $d \neq-2$, and then we check the dimension formula. Recall that for a presentation of the ideal defining the algebra $A$ over a power series ring $P$,

$$
0 \rightarrow R \rightarrow F \rightarrow I \rightarrow 0
$$


and $R_{0} \subset R$ the submodule of Koszul relations, the module $T_{A}^{2}$ is the cokernel of the natural map of $A$-modules

$$
\operatorname{Hom}_{B}\left(F / R_{0} \otimes_{P} A, A\right) \rightarrow \operatorname{Hom}\left(R / R_{0}, A\right) .
$$

By (5.2.1) the generators of $R$ are in degree 3 so that there can be nothing of degree -4 or lower in $T_{A}^{2}$. Also it is easy to check that $\left(T_{A}^{2}\right)_{-3}=0$ (cf. [12, 3.8]) since $A$ is defined by quadratic equations with sufficiently many linear syzygies. The nonnegative part of $T_{A}^{2}$ is zero simply because there are no elements in $A$ of degree 3 or higher. It remains to check the degree -1 . The degree 2 part of $A$ is the one-dimensional socle of $A$, and the injective map $R_{3} \rightarrow F_{3}$ in (2) is changed into its transpose when forming

$$
\operatorname{Hom}_{B}\left(F / R_{0} \otimes_{P} A, A\right)_{-1} \rightarrow \operatorname{Hom}\left(R / R_{0}, A\right)_{-1} .
$$

Hence the cokernel of the latter map is zero.

For degree -2 we first compute $\operatorname{Hom}\left(R / R_{0}, A\right)_{-2}$ : By local duality for the Gorenstein algebra $A$, which has its socle in degree 2 , this homogeneous part is dual to $\left(R / R_{0}\right)_{4}$. The relevant piece of the degree 4 part of the graded resolution is

$$
0 \rightarrow\left(F_{3}\right)_{4} \rightarrow\left(F_{2}\right)_{4} \rightarrow R_{4} \rightarrow 0
$$

and the degree 4 part of $F_{3}$ is spanned by a minimal set of generators of the second syzygies.

Subtracting the number of Koszul relations (minimal generators of $R_{0}$ ) we get

$$
\begin{aligned}
& \operatorname{dim}_{\mathrm{C}}\left(R / R_{0}\right)_{4} \\
& \quad=r^{2}(r+2)(r-2) / 3-r(r-1)(r-3)(r+2) / 8-\left(\begin{array}{c}
(r-1)(r+2) / 2 \\
2
\end{array}\right) \\
& =\left(r^{4}-r^{2}-12\right) / 12 .
\end{aligned}
$$

On the degree -2 level the map (3) is injective because the chosen resolution was minimal. Subtracting the number of equations in $I$ we get

$$
\operatorname{dim}_{\mathbf{C}}\left(T_{A}^{2}\right)_{-2}=r(r+1)(r+2)(r-3) / 12 .
$$

5.4. Now we come back to the cotangent cohomology of elliptic partition curves.

Proposition 5.4.1. For $(Y, 0)$ an elliptic partition curve of multiplicity $n+1$, and with $r$ branches,

1. $\operatorname{dim}_{\mathbf{C}} T_{Y}^{1}=n(n+1) / 2-r+1$.

2. $\operatorname{dim}_{C} T_{Y}^{2}=n(n+1)(n-4) / 6$ and $T_{Y}^{2}$ is annihilated by the maximal ideal of the local ring.

Proof. For (i) we combine the following facts:

- $(Y, 0)$ is a hypersurface section of a simply elliptic singularity of degree $n+1$.

- This simply elliptic singularity has $\operatorname{dim}_{C} T_{X}^{2}=(n+1)(n-4) / 2$, and $T_{X}^{2}$ is annihilated by the maximal ideal of the local ring, because it is concentrated in one homogeneous degree $(-2)[12,3.8]$.

- The dimension of the smoothing components of $Y$ is $\mu=2(n+1)-$ $r+1$. 
Plugging this into the Main Lemma we get our conclusion.

The Main Lemma applied to the Gorenstein fat point of minimal multiplicity $r+2=n+1$ tells us that $T_{Y}^{2}$ is minimally generated by

$$
r(r-1)(r+4) / 6-\left(r^{2}+r+2\right) / 2=n(n+1)(n-4) / 6
$$

elements: the first summand is $\operatorname{dim} T^{1}$ of the fat point, and the second is the dimension of the smoothing component, equal to $\operatorname{dim}_{\mathrm{C}} T^{0}$ by $[3,6.3 .1]$.

By semicontinuity of $\operatorname{dim} T_{Y_{t}}^{2}$ in flat families we are done if we can show that the most special elliptic partition curve-the monomial curve with semigroup $\{0, n+1, \ldots, 2 n, 2 n+2, \ldots\}$-has a $T^{2}$ of dimension $n(n+1)(n-4) / 6$. This follows from a computation which we omit.

\section{Proof of THE MAIN RESUlt}

6.1. In this section we state and prove the main result of this article. If $M$ is a module over a local ring let $\operatorname{cg} M=\operatorname{dim} M / \mathrm{m} M$, i.e. the minimal number of generators of $M$. For a surface singularity $X$ we denote $n+1=\operatorname{dim} \mathbf{m} / \mathbf{m}^{2}$ the embedding dimension. Let, moreover, $\pi: \widetilde{X} \rightarrow X$ be a resolution (usually the minimal resolution), with $E=\bigcup_{i=1}^{k} E_{i}$ the exceptional divisor, and denote the fundamental cycle by $Z$.

Theorem 6.1.1. For a minimally elliptic surface singularity of embedding dimension $n+1 \geq 5$, and $f$ the defining equation of a general hypersurface section,

1. $\operatorname{cg} T_{X}^{2}=(n+1)(n-4) / 2$. Moreover, if $\mathbf{m}_{X}$ is the maximal ideal, then $\mathbf{m}_{X} T_{X}^{2}=f T_{X}^{2}$.

2. If the exceptional curve of the blow up of the maximal ideal is reducedi.e. if $Z$ is reduced on non-(-2) curves-then $\operatorname{dim} T_{X}^{2}=(n+1)(n-4) / 2$.

Remark. The minimally elliptic singularities which fit the hypotheses of (2) are exactly the Kodaira singularities. The exceptional curve on the minimal resolution of such a singularity is isomorphic (as an abstract curve) to a fibre (regular or singular) in a fibration by elliptic curves [7, Theorem 6.2]. In particular the following cases are included:

1. Simply elliptic singularities (type $I_{0}$ ).

2. Cusp singularities (type $\mathbf{I}_{b}, b \geq 1$ ).

3. Triangle singularities and their nonquasihomogeneous satellites (types II, III, and IV).

An example with highly nonreduced fundamental cycle and reduced tangent cone is the series II $^{*}\left(=\widetilde{E}_{8}\right)$ with dual graph of the minimal resolution

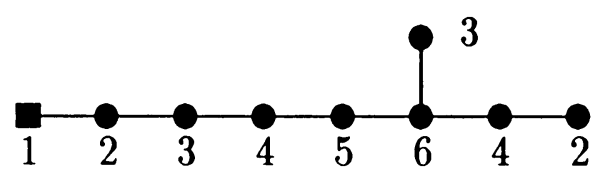

where $\mathbf{~ i s ~ a ~ r a t i o n a l ~ c u r v e ~ o f ~ s e l f - i n t e r s e c t i o n ~ n u m b e r ~}-b, b \geq 3$, and all the other curves are rational of self-intersection number -2 . The numbers indicate the multiplicities of the fundamental cycle. 
6.2. The proof of this theorem is given below. Its structure is analogous to the proof of $[3,5.1 .1]$ but some of the arguments used here are new. Part (1) follows easily from $\S 5$ and the Main Lemma. To prove (2) we actually compute $T^{2}$ of the (nonisolated) singularity at the vertex of the tangent cone of $X$.

Proof. From the Main Lemma and Proposition 5.4.1 we know that

$$
\operatorname{dim}_{\mathbf{C}} T_{X}^{2} / f T_{X}^{2}=(n+1)(n-4) / 2 \text {. }
$$

Moreover, obviously $\operatorname{cg} T_{X}^{2}=\mathrm{cg} T_{X}^{2} / f T_{X}^{2}$. If $Y=f^{-1}(0)$ is the hypersurface section, then by Proposition 5.4.1, $m T_{Y}^{2}=0$. Since $T_{X}^{2} / f T_{X}^{2} \hookrightarrow T_{Y}^{2}$ we get

$$
\operatorname{cg} T_{X}^{2}=\operatorname{cg} T_{X}^{2} / f T_{X}^{2}=\operatorname{dim}_{C} T_{X}^{2} / f T_{X}^{2}=(n+1)(n-4) / 2 .
$$

Now let $X$ satisfy the conditions of the second part of the theorem. Denote the singularity at the vertex of the tangent cone of $X$ by $\bar{X}$. Let $C$ be the projectivized tangent cone. The reduced curve $C$ is isomorphic to the exceptional divisor of the first blow up of $X$. Our assumptions imply that $C$ is a (in general singular) arithmetically Cohen-Macaulay elliptic curve of degree $n+1$ in $\mathbf{P}^{n}$ [11, Proof of 2.8].

The singularities of $C$ are hypersurface sections of singularities of the blow up $\widehat{X}$, cut out by a local equation of the exceptional curve. In particular they are hypersurfaces, and the sheaf $\mathscr{T}_{C}^{2}$ vanishes. Hence $T_{\bar{X}}^{2}$ is supported at the vertex, hence it is of finite dimension.

6.3. Let $T_{\bar{X}}^{2}, k$ be the homogeneous part of degree $k$ in the natural grading of $T_{\bar{X}}^{2}$. By Theorem 5.2.2 of [3] there is an injective map

$$
T_{\bar{X}, k}^{2} \hookrightarrow H^{1}\left(C, \mathscr{N}_{C}(k)\right),
$$

$\mathscr{N}_{C}(k)$ the normal bundle, for all $k$. We use this to prove

Proposition 6.3.1. If $X$ is a minimally elliptic surface singularity with reduced tangent cone $\bar{X}$, then $\operatorname{dim}_{\mathbf{C}} T_{\bar{X}}^{2}=(n+1)(n-4) / 2$ and $T_{\bar{X}}^{2}$ is concentrated in degree -2 .

Proof. As in $[3, \S 5.2 .3]$ the proof is given in 3 steps. Steps (2) and (3) are very similar to the corresponding arguments there.

Step 1. $H^{1}\left(C, \mathscr{N}_{C}(k)\right)=0$ for $k \geq-1$. Consider the exact sequence

$$
0 \rightarrow \boldsymbol{\Theta}_{C}(k) \rightarrow \boldsymbol{\Theta}_{\mathbf{p}^{n}} \otimes \mathscr{O}_{C}(k) \rightarrow \mathscr{N}_{C}(k) \rightarrow \mathscr{T}_{X}^{1}(k) \rightarrow 0 .
$$

Since $C$ is reduced, $\mathscr{T}_{C}^{1}$ has support at points. Therefore $H^{1}\left(C, \mathscr{T}_{C}^{1}(k)\right)=0$, and $H^{1}\left(C, \mathscr{N}_{C}(k)\right)=0$ if $H^{1}\left(C, \Theta_{\mathbf{p}^{n}} \otimes \mathscr{O}_{C}(k)\right)=0$. To compute the latter cohomology group, twist and apply cohomology to the standard restricted Euler sequence

$$
0 \rightarrow \mathscr{O}_{C} \rightarrow \mathscr{O}_{C}(1)^{n+1} \rightarrow \Theta_{\mathbf{p}^{n}} \otimes \mathscr{O}_{C} \rightarrow 0 .
$$

This gives the vanishing of $H^{1}\left(C, \Theta_{\mathbf{p}^{n}} \otimes \mathscr{O}_{C}(k)\right)$ for $k \geq 0$ at once, because the very ample $\mathscr{O}_{C}(k+1)$ has vanishing cohomology. The interesting case is $k=-1$.

The associated long exact cohomology sequence reads

$$
\cdots \rightarrow H^{1}\left(C, \mathscr{O}_{C}(-1)\right) \stackrel{\psi}{\longrightarrow} H^{1}\left(C, \mathscr{O}_{C}\right)^{n+1} \rightarrow H^{1}\left(C, \Theta_{\mathbf{p}^{n}} \otimes \mathscr{O}_{C}(-1)\right) \rightarrow 0 .
$$


We claim that the map $\psi$ is an isomorphism.

To prove this assertion apply Serre duality on $C$. The dualizing module of $C$ is $\mathscr{O}_{C}$ as can be seen, for example, by adjunction formula on $\widehat{X}$. The dual map is

$$
\psi^{*}: H^{0}\left(C, \mathscr{O}_{C}\right)^{n+1} \rightarrow H^{0}\left(C, \mathscr{O}_{C}(1)\right),
$$

and $\psi^{*}$ is the restriction to $C$ of the corresponding map on $\mathbf{P}^{n}$,

$$
H^{0}\left(\mathbf{P}^{n}, \mathscr{O}_{\mathbf{P}^{n}}\right)^{n+1} \rightarrow H^{0}\left(\mathbf{P}^{n}, \mathscr{O}_{\mathbf{P}^{n}}(1)\right) .
$$

By [5, II.8.13] this is the degree-1 piece of the first differential of the Koszul complex on projective $n$-space. It maps the $i$ th basis vector on the left-hand side to the $i$ th homogeneous coordinate $x_{i}$ of $\mathbf{P}^{n}, i=0, \ldots, n$. The curve $C$ is not contained in any hyperplane of $\mathbf{P}^{n}$, so $\psi^{*}$ is injective, and hence surjective because both source and target have the same dimension $n+1$.

Step 2. $T_{\bar{X}, k}^{2}=0$ for $k \leq-3$. This is proved exactly as in $\S 5.2 .3(2)$ of [3].

Step 3. Application of the Main Lemma. Since we know that $T_{X}^{2}$ is concentrated in one degree it is automatically annihilated by the maximal ideal. All we have to do is to compute the corank. But the general hypersurface section is the singularity of $n+1$ lines in $n$-space, which proves the proposition.

To finish the proof, observe that there is a normally flat deformation $\phi$ : $\mathscr{X} \rightarrow(D, 0)$ with a section $\sigma:(D, 0) \rightarrow \mathscr{X}$, and a good representative $\phi:$ $\mathscr{X} \rightarrow D$ such that for $t \in D \backslash\{0\}$ the singularity $\left(\mathscr{X}_{t}, \sigma(t)\right) \cong X$, and $\mathscr{X}_{0} \cong \bar{X}$. Semicontinuity establishes

$$
\operatorname{dim} T_{X}^{2}=(n+1)(n-4) / 2 .
$$

6.4. Theorem 6.1 .1 does not extend to minimally elliptic surface singularities with nonreduced projective tangent cone. The first counterexample follows.

Example. Let $X$ be a singularity of embedding dimension 7 with dual resolution graph

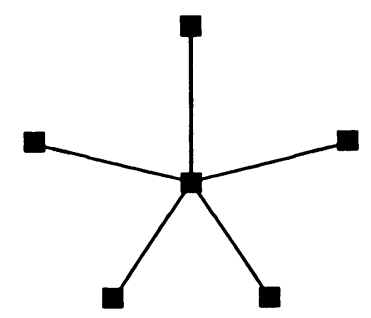

all self-intersection numbers are -3

Such an $X$ can be chosen to be quasihomogeneous with weights $2\left(x_{1}, x_{2}\right)$ and $3\left(x_{3}, \ldots, x_{7}\right)$ for the generators of the affine algebra. In the most symmetric case the equations are

$$
\begin{gathered}
x_{1} x_{4}-x_{2} x_{3}, \quad x_{1} x_{5}-x_{2} x_{4}, \quad x_{1} x_{6}-x_{2} x_{5}, \quad x_{2} x_{7}-x_{2} x_{6}, \\
x_{2} x_{5}-x_{4}^{2}, \quad x_{3} x_{6}-x_{4} x_{5}, \quad x_{3} x_{7}-x_{4} x_{6}, \\
x_{4} x_{6}-x_{5}^{2}, \quad x_{4} x_{7}-x_{5} x_{6}, \quad x_{5} x_{7}-x_{6}^{2} \\
x_{3} x_{4}-x_{5} x_{6}+x_{1}^{3}, \quad x_{4} x_{5}-x_{6} x_{7}+x_{1} x_{2}^{2} \\
x_{4}^{2}-x_{6}^{2}+x_{1}^{2} x_{2}, \quad x_{5}^{2}-x_{7}^{2}+x_{2}^{3} .
\end{gathered}
$$


The fundamental cycle, $Z$, is not reduced; the central curve appears with multiplicity 2. Since $X$ resolves in one blow up, it is clear that the tangent cone is also nonreduced.

Using the computer-algebra program Macaulay, written by D. Bayer and M. Stillman, we find that

$$
\operatorname{dim}_{\mathbf{C}}\left(T_{X}^{1}\right)_{k}= \begin{cases}5, & k=-3, \\ 3, & k=-2, \\ 5, & k=-1, \\ 2, & k=0, \\ 1, & k=2, \\ 0, & \text { otherwise }\end{cases}
$$

which can also be checked by [12], and

$$
\operatorname{dim}_{\mathbf{C}}\left(T^{2}\right)_{k}= \begin{cases}2, & k=-6, \\ 5, & k=-5, \\ 1, & k=-4, \\ 0, & \text { otherwise }\end{cases}
$$

We also checked the obstruction space of the (nonreduced) singularity at the vertex of the affine tangent cone $\bar{X}$. The result was that already there $\operatorname{dim} T_{\bar{X}}^{2}=$ 8 with 7 dimensions in degree -2 as suggested by Proposition 6.3.1, and one additional element in degree $(-1)$. Since exactly the same phenomenon already occurred for our counterexample 5.5 of [3] we would like to raise the following question:

For a rational or minimally elliptic singularity $X$ with $T_{\widehat{X}}^{2}=0$ for $\hat{X}$ the first blow up, is $\operatorname{dim} T_{X}^{2}=\operatorname{dim} T_{\bar{X}}^{2}$ ?

\section{REFERENCES}

1. M. Artin, On isolated rational singularities of surfaces, Amer. J. Math. 88 (1966), 129-136.

2. K. Behnke, On projective resolutions of Frobenius algebras and Gorenstein rings, Math. Ann. 257 (1981), 219-238.

3. K. Behnke and J. Christophersen, Hypersurface sections and obstructions (rational surface singularities), Compositio Math. 77 (1991), 233-258.

4. G. M. Greuel and E. Looijenga, The dimension of smoothing components, Duke Math. J. 52 (1985), 263-275.

5. R. Hartshorne, Algebraic geometry, Graduate Texts in Math., vol. 52, Springer, New York, 1977.

6. H. Hironaka, On the equivalence of embeddings, Arithmetical Algebraic Geometry (Conference Purdue Univ., 1963) (O. F. G. Schilling, ed.), Harper and Row, New York.

7. K. Kodaira, On compact analytic surfaces. II, Ann. of Math. 77 (1963), 563-626.

8. E. Kunz, The value semigroup of a one-dimensional Gorenstein ring, Proc. Amer. Math. Soc. 25 (1970), 748-751.

9. H. Laufer, On minimally elliptic singularities, Amer. J. Math. 99 (1977), 1257-1295.

10. M. Reid, Elliptic Gorenstein singularities of surfaces, Preprint (unpublished). 
11. J. Wahl, Equations defining rational singularities, Ann. Sci. École Norm. Sup. 20 (1977), 231-264.

12. __ The jacobian algebra of a quasihomogeneous Gorenstein surface singularity, Duke Math. J. 55 (1987), 843-871.

Mathematisches Seminar, Bundesstrasse 55, D-2000 Hamburg 13, Germany

Current address: Untere Kellerstr. 47a, D-8501 Feucht.

E-mail address: pla_kbh@pki_nbg.philips.de

Matematisk Institutt, PB 1053 Blindern, 0316 Oslo 3, Norway

E-mail address: christop@ma-mail.uio.no 